\title{
Amino acid transporter as an osmo-regulator in aquatic organisms
}

\author{
HARUHTKO TOYOHARA AND KAZUHARU TAKEUCHI \\ Division of Applied Biosciences, Graduate School of Agriculture, Kyoto University, Kitashirakawa-oiwakecho, \\ Sakyo, Kyoto 606-8502, Japan (toyohara@kais.kyoto-u.ac.jp)
}

SUMMARY: Marine organisms including fish (vertebrate) and shellfish (invertebrate) adapt to changes in the osmolality at the cellular level. Fish can maintain the osmolality of body fluid within a certain range due to the highly developed endocrinological system, while shellfish cannot regulate the osmolality of body fluid due to the lack of it. This suggests that shellfish cells are more resistant against osmotic change than fish cells. To compare the osmo-responsive system of fish and shellfish at cellular level, we focussed on the amino acid transporters and examined the expression patterns of their mRNAs following the osmotic change. When examined the expression patterns of fish taurine transporter $m R N A$ following the osmotic change by using EPC cells, cultured carp epidermal cells, specific induction by hyper-osmotic stress was observed. Hyper-osmolality induced expression was also observed in tilapia tissues when tilapia acclimatized to fresh water was immediately exposed to $70 \%$ seawater. In contrast to fish, mRNA of oyster amino acid transporter was induced both by hyper- and hypo-osmotic stresses. Unexpected induction of oyster transporter mRNA by hypo-osmotic stress suggests a difference in the promoter function of the transporter genes between fish and shellfish.

\section{KEY WORDS: amino acid, carp, osmolality, oyster, taurine, tilapia, transporter}

\section{INTRODUCTION}

Marine organisms must cope with a variety of stresses such as changes in temperature, oxygen concentration, and osmolality in addition to ultraviolet and pollutants. In the present review, we focus on the cellular mechanism of osmotic adaptation of fish and shellfish, because marked difference is expected between them due to the difference in dependency on the endocrinological system to regulate the osmolality of body fluid.

\section{OSMOTIC ADAPTATION AT CELLULAR LEVEL}

As shown in Fig.1, diadromous fish such as salmon and eel can live both in fresh water and seawater. In addition to the diadromous fish, fresh water fish such as carp can live in brackish water if they are gradually acclimatized. Similarly, seawater fish such as flatfish and black sea bream can live in brackish water by gradual acclimatization. Such high adaptability of fish against osmotic change is due to the well-developed endocrinological system. ${ }^{1)}$ According to the change in the environmental salinity, osmolality of the body fluid of fish changes within certain range, suggesting that cells other than epithelial cells are exposed to the osmotic change in the course of migration from fresh water to seawater or seawater to fresh water.

When cell growth under various osmolality was compared, most fish cell lines demonstrated greater resistance against osmotic change than mammalian cell lines, suggesting fish cells are equipped with specific molecular system to adapt to osmotic change. As a matter of fact, environmental osmotic change induced the expression of specific proteins in carp (Cyprinus carpio) epidermal cells, $\mathrm{EPC}^{2)}$ It is of interest that the threefold of the osmolality to induce apoptic cell death could be elevated by gradual acclimatization. ${ }^{3,4)}$ These findings suggested fish cells adapt to change in environmental osmolality by expressing as yet unidentified osmo-responsive genes although not unidentified.

Shellfish living in estuaries are also exposed against to change according to the tidal change or heavy rainfall, because most shellfish have no or little mobile ability. Due to the lack of endocrinological system to maintain the osmolality of body fluid constant, cells of shellfish are expected to be more resistant against osmotic change than fish cells. However, no direct evidence of the osmotic resistance of shellfish cells has been demonstrated, because no cell lines are available to study the osmotic resistance at cellular level. 


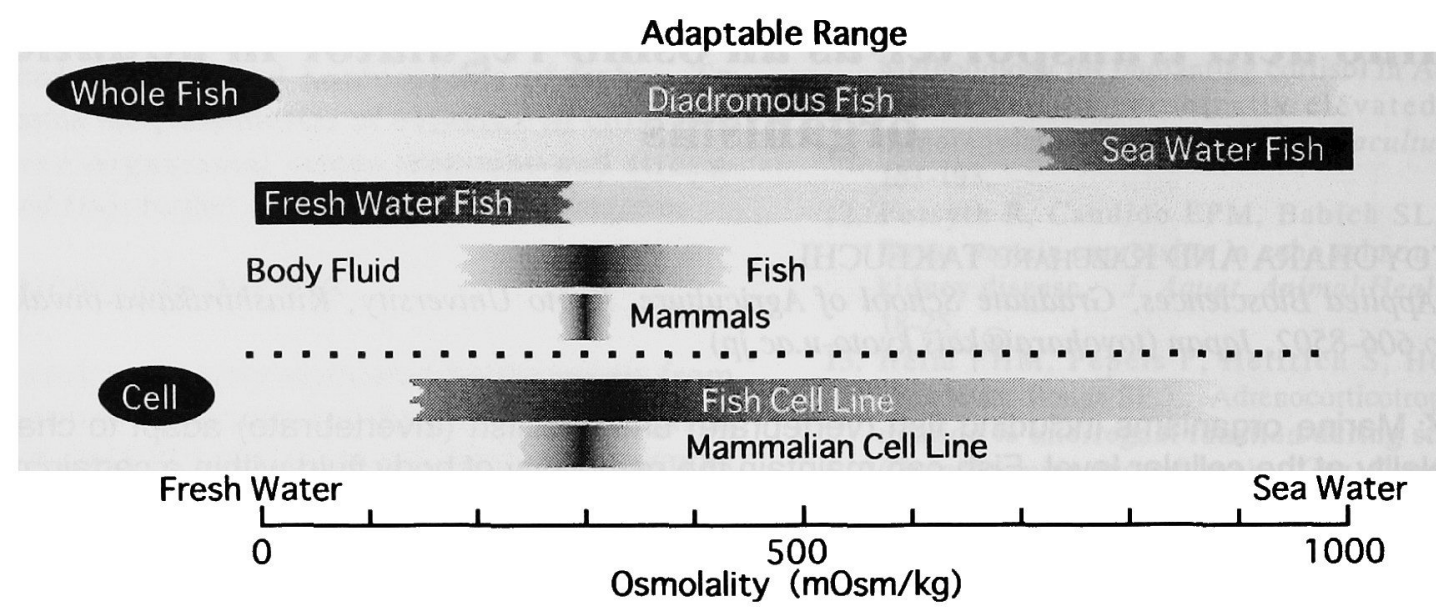

Fig.1 Comparison of osmotic tolerance between fish and mammals at the cellular level.

\section{AMINO ACID TRANSPORTER AS AN HYPER- OSMOLALITY INDUCED GENE}

To identify the osmo-responsive genes, we attempted to clone the genes specifically induced by osmotic change and successfully identified one hyper-osmotic responsive gene from EPC cells. ${ }^{5)}$ The deduced amino acid sequence of the cDNA predicts that the cDNA encodes a $\mathrm{Na}^{+}, \mathrm{Cl}^{-}$-dependent taurine transporter having a twelve transmembrane spanning structure. As a matter of fact, COS-7 cells transfected by the cloned cDNA expressed the taurine uptake activity, suggesting the cDNA encodes a functional taurine transporter. Taurine (2-aminoethane sulfonic acid) is most abundant osmolites in fish cells, suggesting that taurine transporter is likely to play an important role in the acclimatization against hyperosmotic condition by uptaking taurine and maintaining intracellular osmolality high.

Then we attempted to clone a cDNA encoding an osmo-responsive amino acid transporter from oyster (Crassostrea gigas) and obtained a transporter cDNA of which translated product showed a twelve transmembrane spanning structure and demonstrated the uptaking activity on various amino acid according to the increase in extracellular $\mathrm{Na}^{+}$and $\mathrm{Cl}^{-}$ concentration when transfected in COS-7 cells (Toyohara et al. Unpubl.data 2001).

\section{COMPARISON OF EXPRESSION MANNER OF THE TRANSPORTER GENES BETWEEN FISH AND SHELLFISH}

By the northern blot analysis, the expression of taurine transporter mRNA was induced only by hyper-osmotic stress nor by hypo-osmotic stress in
EPC cells. To elucidate the expression of mRNA at the whole fish level, we examined the expression of its mRNA in various tissues of tilapia (Oreochromis mossambicus) when fish acclimatized with fresh water were immediately transferred to $70 \%$ seawater. ${ }^{6)}$ As a result, all tissues tested demonstrated the elevated expression of mRNA of taurine transporter, suggesting the importance of taurine transporter in hyper-osmotic acclimatization at the whole fish level.

As for oyster transporter, however, the expression of mRNA was observed both by hyperand hypo-osmotic stresses (Toyohara et al. Unpubl.data 2001). Interestingly, more markedly elevated expression was detected by hypo-osmotic stress than hyper-osmotic stress. Theoretically, mRNA expression level of transporter is expected to up-regulate only by hyper-osmotic stress due to its role to increase intracellular amino acid concentration. Thus, the unexpected induction of the transporter mRNA in oyster by hypo-osmotic stress could be explained by assuming that the transporter plays a role to recover amino acids which were once released from cells for the adaptation against hypo-osmotic condition.

A putative mechanism to regulate cell volume constant under hyper- and hypo-osmotic conditions is summarized in Fig.2. When exposed against hyperosmotic condition, cell shrinks within a few seconds by water outflow, but cell volume decrease is harmful for the cell. Thus, the cell recovers an initial volume by importing various ions within a few minutes and increases intracellular osmolality. However, high concentration of these ions especially $\mathrm{Na}^{+}$impairs the cellular function. Therefore, the cell of fish and shellfish newly expresses amino acid transporters on the membrane within a few hours or a few days and 


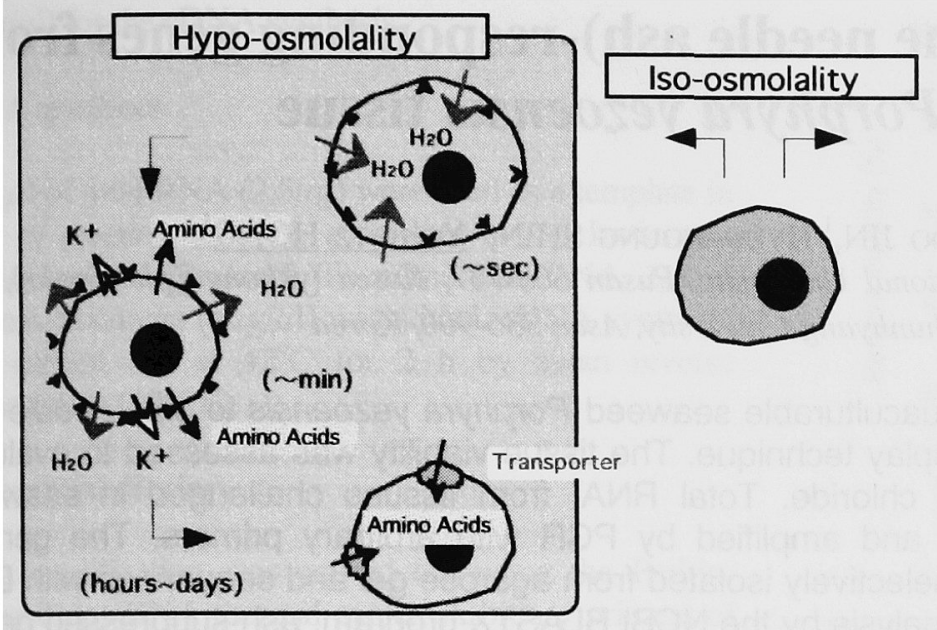

Shellfish

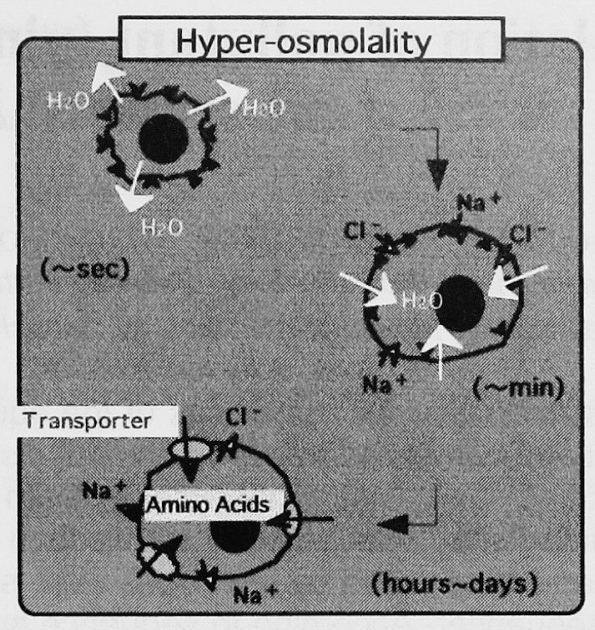

Fish and Shellfish

Fig.2 Comparison of the expression manner of amino acid transporter responding hyper- and hypo-osmotic conditions between fish and shellfish cells.

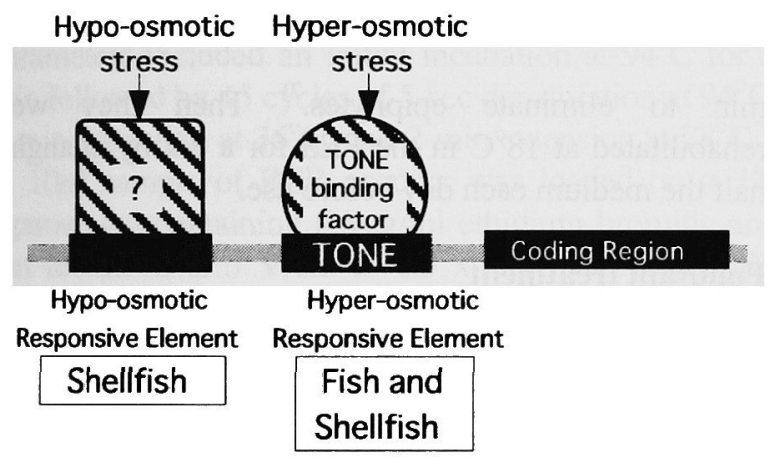

Fig.3 Putative osmo-responsive elements in fish and shellfish amino acid transporter genes.

imports extracellular amino acids instead of exporting ions.

On the contrary, when exposed against hypoosmotic condition, cell swells within a few seconds by water inflow, but cell volume increase is harmful for the cell. Thus, the cell exports $\mathrm{K}^{+}$and amino acids outside with water to decrease intracellular osmolality. Only shellfish cell expresses amino acid transporters within a few hours or a few days possibly to recover amino acids. It remains unknown why fish cell does not express amino acid transporters under hypo-osmotic condition.

As shown in Fig.3, fish taurine transporter gene is supposed to have an element sequence called TONE in the promoter region which is know to have a function inducing the transcription of mRNA in response to the hyper-osmotic stress. ${ }^{7)}$ In case of oyster transporter gene, existence of unidentified hypo-osmotic responsive element is expected in the promoter region in addition to TONE, because the expression of mRNA is elevated by hypo-osmotic stress as well hyper-osmotic stress. We are now trying to clone promoter region of oyster amino acid transporter gene to identify the hypo-osmolality responsive element.

\section{REFERENCES}

1. Bentley PJ. Endocrines and Osmoregulation, SpringerVerlag, Berlin, Heidelberg, New York. 1971.

2. Takeuchi K, Toyohara H, Sakaguchi M. Effect of hyperand hypoosmotic stress on protein synthesis in cultured epidermal cells of common carp. Fisheries Sci. 2000; 66: 117-123.

3. Hashimoto $\mathrm{H}$, Takeuchi $\mathrm{K}$, Matsuo $\mathrm{Y}$, Toyohara $\mathrm{H}$, Sakaguchi M. Induction of apoptosis in fish cells by Hashimoto H, Matsuo Y, Toyohara H, Sakaguchi M. Suppression of apoptosis by acclimatization of fish cells to hypertonicity. Fisheries Sci. 1998; 65: 144-148.

4. Takeuchi K, Toyohara H, Sakaguchi M. A Hyperosmotic-stress-induced mRNA of carp cell encodes $\mathrm{Na}^{+}$and $\mathrm{Cl}^{-}$-dependent high affinity taurine. Biochem. Biophys. Acta. 2000; 1464: 219-230.

5. Takeuchi K, Toyohara H, Kinoshita M, Sakaguchi M. Ubiquitous increase in taurine transporter mRNA in tissues of tilapia (Oreochromis mossambicus) during high salinity adaptation. Fish. Physiol. Biochem. 2001; 23: 173-182.

6. Takenaka M, Preston AS, Kwon HM, Handler JS. The tonicity-sensitive element that mediates increased transcription of the betaine transporter gene in response to hypertonic stress. J.Biol.Chem. 1994; 269: 2937929381. 